\section{In Vivo Percutaneous} Penetration/Absorption, Washington,

\section{D.C., May 1989}

Vinod P. Shah, ${ }^{1}$ Gordon L. Flynn, ${ }^{2}$ Richard H. Guy, ${ }^{3}$ Howard I. Maibach, ${ }^{4}$ Hans Schaefer, ${ }^{5}$ Jerome P. Skelly, ${ }^{1}{ }^{\text {Ronald C. Wester, }}{ }^{3}$ and Avraham Yacobi ${ }^{6}$

\section{CONTRIBUTORS}

Bradley D. Anderson, ${ }^{7}$ Klaus E. Andersen, ${ }^{8}$ Brian W. Barry, ${ }^{9}$ Charan R. Behl, ${ }^{10}$ Leslie Z. Benet, ${ }^{3}$ Robert L. Bronaugh, ${ }^{11}$ Daniel A. W. Bucks, ${ }^{3}$ Annette L. Bunge, ${ }^{12}$ Yie W. Chien, ${ }^{13}$ C. Carnot Evans, ${ }^{1}$ Gordon L. Flynn, ${ }^{2}$ Thomas J. Franz, ${ }^{14}$ William R. Good, ${ }^{15}$ Richard H. Guy, ${ }^{3}$ William I. Higuchi, ${ }^{7}$ Robert S. Langer, ${ }^{16}$ Howard I. Maibach, ${ }^{4}$ JeanPaul Marty, ${ }^{17}$ Gabriela Nicolau, ${ }^{6}$ Esther Patrick, ${ }^{4}$ Carl C. Peck, ${ }^{1}$ Lynn K. Pershing, ${ }^{7}$ Virgil A. Place, ${ }^{18}$ Boyd J. Poulsen, ${ }^{19}$ Jim E. Riviere, ${ }^{20}$ Andre Rougier, ${ }^{21}$ Hans Schaefer, ${ }^{5}$ Vinod P. Shah, ${ }^{1}$ Jane E. Shaw, ${ }^{18}$ Jerome P. Skelly, ${ }^{1}$ Solomon Sobel, ${ }^{1}$ Richard Stoughton, ${ }^{22}$ and Ronald C. Wester ${ }^{3}$

The workshop, held in Washington, D.C., May 1-3, 1989, was sponsored by the American Association of Pharmaceutical Scientists, U.S. Food and Drug Administration, American Academy of Dermatology, Skin Pharmacology Society, and U.S. Army Environmental Hygiene Agency.

${ }^{1}$ Center for Drug Evaluation and Research, Food and Drug Administration, Rockville, Maryland 20857.

${ }^{2}$ University of Michigan, College of Pharmacy, Ann Arbor, Michigan 48109.

${ }^{3}$ University of California, School of Pharmacy, San Francisco, California 94143.

${ }^{4}$ University of California, Department of Dermatology, San Francisco, California 94143.

${ }^{5}$ Centre International de Recherches Dermatologiques, Sophia Antipolis, 06565 Valbonne Cedex, France.

${ }^{6}$ American Cyanamid Company, Pearl River, New York 10965.

${ }^{7}$ University of Utah, Department of Medicine, Division of Dermatology, Salt Lake City, Utah 84132.

${ }^{8}$ Department of Dermatology, Odense University Hospital, Odense, Denmark.

${ }^{9}$ University of Bradford, School of Pharmacy, West Yorkshire, BD71DP Bradford, England.

${ }^{10}$ Hoffman-La Roche, Nutley, New Jersey 07110.

${ }^{11}$ Center for Food Safety and Nutrition, FDA, Washington, DC 20204.

${ }^{12}$ Colorado School of Mines, Chemical Engineering and Petroleum Refining Department, Golden, Colorado 80401.

${ }^{13}$ University of Rutgers, Controlled Drug Delivery Research Center, Piscataway, New Jersey 08855.

${ }^{14}$ University of Arkansas for Medical Sciences, Department of Dermatology, Little Rock, Arkansas 72205.

${ }^{15}$ Ciba-Geigy Corporation, Ardsley, New York 10502.

${ }^{16}$ Massachusetts Institute of Technology, Cambridge, Massachusetts 02139 .

${ }^{17}$ University de Paris-Sud, Faculte de Pharmacie, 92290 ChatenayMalabry, Cedex, France.

\section{OBJECTIVES}

This workshop, "In Vivo Percutaneous Penetration/Absorption," was held in Washington, D.C., on May $1-3,1989$. The first workshop in this series, "In Vitro Percutaneous Penetration," took place in November 1986 (the report of this earlier meeting was published in Pharmaceutical Research, Vol. 4, pp. 265-267, 1987).

The objectives of the workshop were to review the relevant literature and to address the following in detail:

- In vivo percutaneous penetration/absorption methodology.

- The characteristics of dosage forms designed for application to the skin.

- Critical factors controlling in vivo drug transport into and across the skin.

- The use of models in the assessment and evaluation of in vivo percutaneous penetration/absorption.

- Bioavailability/bioequivalence considerations for topical drug products.

Scientific knowledge and technology are rapidly evolving in the topical and transdermal drug products area. This report focuses on the methodologies available for the measurement of percutaneous penetration in vivo; each scientific approach is discussed briefly, followed by advantages and disadvantages of the methodology.

\section{INTRODUCTION}

Drug products applied to the skin can be subdivided into two categories:

(1) dermatological formulations (creams, ointments, gels, lotions) intended for the treatment of local (i.e., application site) skin disorders and

(2) transdermal delivery systems (ointments and patches) intended for the treatment or prevention of systemic disease.

The skin is a barrier to the absorption of topically administered drugs (1-3). As a result, the rate of percutaneous transport is typically slow, and the extent of drug delivery, although concentrated in the skin beneath the application site, is usually low. One advantage of these features is that the incidence of systemic toxicity in topical therapy is far less than that encountered with systemic routes of drug delivery (e.g., oral). Still, topical therapy may fall short of delivering a clinically sufficient local concentration of drug. In such an instance, drug penetration/absorption/bioavailability can be increased through the use of chemical enhancers or through physical enhancing techniques such as iontophoresis and ultrasound. A general problem in ascertaining bioavailability/bioequivalence of topical drug ther-

${ }^{18}$ Alza Corporation, Palo Alto, California 94303.

${ }^{19}$ Syntex Research, Palo Alto, California 94303.

${ }^{20}$ North Carolina State University, College of Veterinary Medicine, Raleigh, North Carolina 27606.

${ }^{21}$ L'Oreal Laboratories de Recherche Fondamentale, Aulnay Sous Bois, France.

${ }^{22}$ University of California at San Diego, Department of Dermatology, La Jolla, California 92093. 
apy is that quantification of the drug in the body (skin and/or systemic circulation) is difficult because the absolute amounts of drug present are (generally) too small. In vivo drug penetration studies following topical application may clarify the poorly resolved bioavailability/bioequivalence issues.

The specific aims of in vivo skin penetration studies may be summarized as follows:

(a) to verify and quantify the cutaneous bioavailability of a topically applied drug,

(b) to verify and quantify the systemic bioavailability of a transdermally delivered drug,

(c) to establish the bioequivalence of different topical formulations of the same drug, and

(d) to determine the incidence of and, if necessary, to quantitate local and systemic toxicological risk following the topical application of a specific drug.

Each of the in vivo percutaneous absorption/penetration approaches discussed at the workshop is now considered in turn. Typically, following an introductory outline of the method, the consensus advantages and drawbacks are listed. Finally, our conclusions are summarized, and future research directions are highlighted.

\section{ANIMAL MODELS}

It is important to emphasize at the outset that the most relevant in vivo data on percutaneous absorption in man will be obtained from studies in humans themselves (4). However, animal models are needed for the development of conceptual insights and to investigate mechanisms. If the study objective is prediction of percutaneous absorption in man, then rate and extent of skin absorption in the animal should be (i) quantitatively the same as in man or (ii) consistently related to the absorption in man by a constant ratio. Moreover, the animals chosen for the studies must respond to treatments (e.g., vehicle effects, especially the use of enhancers) in the same way and to roughly the same degree as in man $(5,6)$. On the basis of the currently available data, the only animals in which permeation data are consistently qualitatively and quantitatively similar to human permeation data are the pig (7) (particularly the weanling pig), the rhesus monkey (8), and the hairless rat (9). However, it should be noted that the extra body fat on the pig may alter drug distribution relative to that in man and thereby confound the results. In the case of the rhesus monkey, skin applications should be limited to the nonhairy regions on the ventral surfaces of the animal. Regional variation in skin properties (thickness, composition, etc.) in animal and man should be considered. Absorption studies in the guinea pig are sometimes predictive of results in man but skin absorption rates in the rabbit, rat, and mouse appears to be substantially greater than that in man $(7,10)$. Furthermore, some laboratory animals do not consistently respond to treatments (e.g., with an absorption enhancer) in the same fashion as man; rather, they seem to be considerably more responsive to such treatments (6).

\section{COLLECTION OF PERMEATED DRUG IN BLOOD, URINE, AND TISSUES}

General. The drug penetration/absorption studies per- formed should differentiate (i) dermatological, topical drug products from transdermal therapeutic drug products, (ii) toxicologic studies from toxicokinetic studies, and (iii) developmental studies from clinical studies. All techniques must consider the benefits of using radiolabel versus nonradiolabeled analytical (e.g., HPLC) methodology.

Four approaches, which sample drug levels in (a) the skin, (b) the venous blood draining the application site, (c) the systemic circulation, and (d) the excreta, can be identified. Principles of pharmacokinetics can be used when the drug concentrations are followed over time. For example, coupled with clearance parameters measured from intraveneous administration, such data can be used to estimate absolute extent of systemic bioavailability of a topically or transdermally applied drug. Using this methodology, drug application to both normal and pathological skin can be considered. For topical dermatological products, toxicokinetic and safety studies should, when possible, be performed in the same species.

\section{SKIN SECTIONING}

Definition. Skin sectioning is cutting, stripping, or otherwise separating skin into its constituent layers for independent assay of their drug content $(11,12)$.

Advantages. The technique can establish distribution and disposition of a given compound in the tissue as a function of time and tissue depth following application under various conditions (different vehicles, diseased skin, etc.). The procedure is best performed in vivo but can, in welldefined circumstances, be conducted in vitro as well.

Disadvantages. Skin sectioning cannot normally be performed in vivo in humans unless limited to punch biopsies. If the experiments are performed in vitro, their relevance is greatest for short application times. The technique is generally limited to radiolabeled studies. The samples collected from the treated skin site in the experiments do not indicate the drug target or the ultimate fate of the drug in the body. Assessments of metabolic processes and metabolites are especially difficult (13).

\section{SAMPLING OF EXCRETA}

Definition. This technique comprises sampling of urine, feces, and expired air for drug content following a topical administration (4).

Advantages. This is useful to assess total absorption. It is relatively noninvasive and can be routinely performed in human subjects. Mass balance of the applied dose must be performed (14).

Disadvantages. A control study involving parenteral injection of the drug is required. Availability of a radiolabeled drug or a sensitive chemical assay is necessary. Metabolism of the drug by the skin and/or systemic metabolism may confound precise bioavailability measurements. The technique has limitations for drugs which are naturally present in the body (e.g., certain steroids). The approach is difficult to apply to fat-soluble drugs with long elimination half-lives.

\section{BLOOD SAMPLING}

Definition. Periodic sampling of blood is routinely performed in all standard pharmacokinetic analyses. 
Advantages. For drugs delivered transdermally to elicit systemic pharmacological effect, assay of drug levels in the blood/plasma is essential. Drug concentrations on the order of those achieved by other administration routes are anticipated. A highly specific and sensitive assay may be necessary permitting high-quality pharmacokinetic data to be obtained. Parent drug and metabolites can be identified and quantitated. With an intravenous "control," absolute bioavailability can be determined.

Disadvantages. The method is generally unsuited for assessment of locally active dermatologic preparations because relationships between systemic and locally effective levels are unclear. The blood concentrations are typically too low for bioavailability assessment. Interference by endogenous substances can also prove problematic.

\section{SKIN FLAP MODELS}

Definition. In these techniques, tissue is surgically prepared so that the blood vessels nourishing and draining the tissue can be directly accessed. Xenografts (human skin transplanted onto an animal model) may be involved $(7,15,16)$.

Advantages. The skin flap models are useful for studies addressing topical drug bioavailability, transdermal delivery, and toxicological/toxicokinetic evaluation. The technique allows assessment of mechanistic questions (e.g., dermal metabolism, cutaneous distribution, effect of blood flow on percutaneous absorption) not easily addressed by other approaches.

Disadvantages. The skin flap model is relatively expensive and, at this time, technically demanding and laborintensive. Animals on which skin flaps with xenografts are raised must be treated systemically with immunosuppressive drugs (e.g., cyclosporine); the effect of this treatment on skin barrier function is unclear.

\section{RESIDUAL ANALYSIS—“DIFFERENCE," "DISAPPEARANCE" METHODS}

\section{Definition}

These are techniques in which the amount of drug absorbed into the skin is assessed as the difference between the amount applied and that recovered at a subsequent time.

Two approaches have been described. (a) After drug application for a fixed time, the residual formulation is washed from the skin surface, and the amount removed is analyzed (17). This is a single-point determination [amount absorbed $=$ (amount applied) - (amount remaining) $]$. (b) The formulation is applied and drug content in the outer skin layers is then followed as a function of time by spectroscopic (e.g., infrared) or radioisotopic monitoring techniques $(18,19)$.

\section{Approach a: Single-Point Measurements of Drug Disappearance}

\section{Advantages}

The procedure requires very small amounts of active formulation. Skin toxicology concerns, while present, are minimal due to the limited exposure (drug amount and skin area). The method is inexpensive and relatively rapid. It is suitable for clinical studies. Radioisotope use is reasonable, due to very low levels required; the radioisotopes need not have high specific activities; very little isotope is consumed.

\section{Disadvantages}

Only one assay per site per application is possible; full characterization of the drug uptake profile requires multiplesite studies. Uniform recovery from different sites must be demonstrated. Drug removed inadvertently from the surface (on clothing, etc.) or by evaporation may be counted as absorbed. For poorly penetrating compounds, the method quantitates the small difference between two large numbers. The application technique is critical; the amount applied and uniformity of spreading must be validated and reproducible. The method does not lend itself to theoretical analysis (separation of nonstationary-state from steady- or quasisteady-state periods of diffusion is virtually impossible). Another potential problem is that the material used (soap solution, solvent, etc.) to remove the remaining preparation from the skin surface may influence drug penetration. Furthermore, the procedure does not actually measure the amount of drug at the target tissue in the skin. In other words, this type of measurement fails to reflect diffusive migration of drug into the critical zone, i.e., the region where the pharmacodynamic, pharmacological, or biochemical event of relevance takes place.

\section{Approach b: Continuous or Periodic Monitoring of Drug Uptake}

The drug, radiolabeled (preferably) with ${ }^{14} \mathrm{C}$ or containing a nonambiguous spectrophotometric marker, is applied to the skin surface in the test formulation. Over time, the disappearance of radioactivity, or of spectral signal, is monitored using, for example, an appropriate Geiger-Muller tube or attenuated total reflectance, Fourier transform infrared spectroscopy (ATR-FTIR). Penetration kinetics are assessed from the decay of the respective signal.

\section{Advantages}

Pharmacologically insignificant drug doses can be used. Full characterization of the drug uptake profile from a single experiment is possible. The method is relatively sensitive. The methods are noninvasive; they are also precise and objective.

\section{Disadvantages}

Radiometric Methods. The use of radioisotopes on human subjects is necessary. The application of the method for bioequivalence measurements has not been demonstrated. Again, one is not measuring drug levels at the target site.

Spectrophotometric Methods. Spectrophotometric interference is a major problem. Optimal application of the technique requires that the drug have one or more unique spectral features that distinguish it from the spectral characteristics of the skin. However, providing the drug with selected carbon-deuterium substitutions (for regular $\mathrm{C}-\mathrm{H}$ bonds) could generalize the approach. The equipment is spe- 
cialized and costly. As with radioisotope monitoring, one is not measuring drug levels at the target site.

\section{IN VIVO MEASUREMENT OF DRUG CONCENTRATIONS IN STRATUM CORNEUM}

\section{Prediction of Penetration}

Definition. This is a technique in which the permeability of drug is projected from the amount recovered in the stratum corneum by adhesive tape-stripping at a fixed time following drug application (20). The method depends upon a correlation between short-time uptake by the stratum corneum and total percutaneous absorption.

Advantages. The method requires pharmacologically insignificant drug doses; the experiment is straightforward and inexpensive; radiolabeled drugs are not essential if the compound can be efficiently extracted from the tape-strips for conventional analysis; comparisons between formulations are easily performed and can be well controlled.

Disadvantages. Quantification of drugs in the stratum corneum tape-strips has generally been limited to radioisotope counting. Other approaches have yet to be optimized and validated. The correlation between the amount of drug in the stratum corneum and the total drug absorption has been established only for some drugs and formulations. Since different body sites of skin have different drug penetration properties, the site of application has to be specified for predicting drug absorption as for any other method. The method does not sample the epidermis or the dermis (i.e., the normal "targets" of topical drug products). The cleaning and preparation of the skin for stripping is a critical determinant of drug recovery.

\section{Mathematical Models in the Assessment of in Vivo Percutaneous Penetration/Absorption}

Mathematical (mechanistic) models of percutaneous penetration have been utilized to simulate drug delivery from the formulation, drug movement through skin into the cutaneous circulation, and subsequent systemic distribution, metabolism, and excretion (21-23). Using appropriate parameters, this approach may lead to experimentation with novel systems. Mathematical models of percutaneous absorption have been developed to extrapolate in vitro measurements to in vivo data, to test mechanistic hypotheses, and to interpret data.

\section{Bioavailability/Bioequivalence Considerations}

For topical dermatological products, a measurable pharmacodynamic response and/or quantification of the amount of drug absorbed/penetrated at the target site can be regarded as a measure of drug availability (bioavailability) from the dosage form $(24,25)$. On the other hand, for transdermal drug products, measurement of systemic drug concentrations and/or pharmacodynamic response provides the necessary evidence of drug bioavailability.

Cutaneous bioavailability can be assessed by performing, as a function of time,
(1) pharmacokinetic measurements of the drüg concentrations in the skin or systemic circulation or

(2) pharmacodynamic measurements of the pharmacological response to the drug in the skin or elsewhere.

The combination of the two methods allows one to investigate problems of principal importance and subsequently to choose one of the two approaches for more routine purposes.

\section{PHARMACODYNAMIC RESPONSES FOR ASSESSING BIOAVAILABILITY AND BIOEQUIVALENCE OF TOPICAL DERMATOLOGICAL PRODUCTS}

Pharmacodynamic responses in skin function can serve as proof, and as measures, of local absorption. To date, vascular responses have been used in this way. The pharmacodynamic assays are worth pursuing (in particular, the skin blanching assay for glucocorticoids) but require development and generation of a rigid worldwide protocol before they can be used for bioavailability/bioequivalence determinations with confidence $(1,26)$.

Advantages. There is a long history with these assays (in particular, the vasoconstriction or skin blanching assay) in dermatological research with glucocorticoids and there are good clinical efficacy correlations. The methods are internally consistent, and for clinical studies, they are inexpensive and facile. The assays are qualitatively reliable and have proven useful for evaluating vehicle effects on drug absorption.

Disadvantages. Only a limited number of compounds (e.g., topical corticosteroids) evoke strong local pharmacological response and lend themselves to the pharmacodynamic method. Pharmacodynamic responses are difficult to validate. The methods require a rigid protocol. The pharmacodynamic measurements are subjective, and there is a need, therefore, to develop objective methods to quantify these pharmacodynamic responses (27). For most topical drug products, the dose-response curves are yet to be defined. The "area under the curve", (AUC) (response versus time) type of measurements and calculations are a necessary part of the evaluation.

\section{BIOAVAILABILITY/BIOEQUIVALENCE OF TRANSDERMAL DELIVERY SYSTEMS}

Pharmacokinetic and pharmacodynamic studies are required as appropriate for innovator and generic drug products which are designed to elicit systemic pharmacological effect. Special attention should be paid to the occurrence and severity of local (i.e., application site) skin reactions. Appropriate irritation and sensitization studies must be performed. Although these requirements are expensive and time-consuming, they are essential. Since transdermal drug products are administered for systemic effects, it is desirable to measure drug/metabolite concentrations in blood/plasma and/or urine.

\section{REFERENCES}

1. B. W. Barry. Dermatological Formulations, Percutaneous Absorption, Marcel Dekker, New York, 1983. 
2. H. Schaefer, A. Zesch, and G. Stuttgen. Skin Permeability, Springer Verlag, Berlin, 1982.

3. R. Bronaugh, and H. I. Maibach. Percutaneous Absorption: Mechanism, Methodology, Drug Delivery, 2nd ed., Marcel Dekker, New York, 1989.

4. R. H. Guy, J. Hadgraft, R. S. Hinz, K. V. Roskos, and D. A. W. Bucks. In vivo evaluations of transdermal drug delivery. In Y. W. Chien (ed.), Transdermal Controlled Systemic Medications, Marcel Dekker, New York, 1987, pp. 179-224.

5. H.-K. Choi, G. L. Flynn, and G. L. Amidon. Transdermal delivery of bioactive peptides: The effect of $n$-decylmethyl sulfoxide, $\mathrm{pH}$, and inhibitors on enkephalin metabolism and transport. Pharm. Res. 7:1099-1106 (1990).

6. J. R. Bond and B. W. Barry. Hairless mouse skin is limited as a model for assessing the effects of penetration enhancers in human skin. J. Invest. Dermatol. 90:810-813 (1988).

7. W. G. Reifenrath, E. M. Chellquist, E. A. Shipwash, W. W. Jederberg, and G. G. Krueger. Percutaneous penetration in the hairless dog, weanling pig, and the grafted athymic nude mouse: Evaluation of models for predicting skin penetration in man. $\mathrm{Br}$. J. Dermatol. 111 (Suppl. 27):123-135 (1984).

8. R. C. Wester and H. I. Maibach. In vivo animal models for percutaneous absorption. In R. L. Bronaugh and H. I. Maibach (eds.), Percutaneous Absorption. Mechanisms-Methodology-Drug Delivery, Marcel Dekker, New York, 1989, pp. 221238.

9. A. Rougier, C. Lotte, and H. I. Maibach. The hairless rat: A relevant animal model to predict in vivo percutaneous absorption in humans? J. Invest. Dermatol. 88:577-581 (1987).

10. M. J. Bartek, J. A. La Budde, and H. I. Maibach. Skin permeability in vivo: Comparison in rat, rabbit, pig and man. J. Invest. Dermatol. 58:114-123 (1972).

11. H. Schaefer, G. Stuttgen, A. Zesch, W. Schalla, and J. Gazith. Quantitative determination of percutaneous absorption of radiolabeled drugs in vitro and in vivo by human skin. In J. H. W. Mali (ed.), Current Problems in Dermatology, Vol. 7, Karger, Basel, 1978, pp. 80-94.

12. H. Schaefer and E. Lamaud. Standardization of experimental models. In B. Shroot and H. Schaefer (eds.), Skin Pharmacokinetics, Karger, Basel, 1987, pp. 77-80.

13. D. B. Guzek, A. H. Kennedy, S. C. McNeill, E. Wakshull, and R. O. Potts. Transdermal drug transport and metabolism. I. Comparison of in vitro and in vivo results. Pharm. Res. 6:33-39 (1989)

14. D. A. W. Bucks, J. R. McMaster, H. I. Maibach, and R. H. Guy. Bioavailability of topically administered steroids: A "mass balance" technique. J. Invest. Dermatol. 91:29-33 (1988).

15. Z. Wojciechowski, L. K. Pershing, S. Huether, L. Leonard, S. A. Burton, W. I. Higuchi, and G. G. Krueger. An experimental skin sandwich flap on an independent vascular supply for the study of percutaneous absorption. J. Invest. Dermatol. 88:439-446 (1987).

16. P. L. Williams, M. P. Carver, and J. E. Riviere. A physiologi- cally relevant pharmacokinetic model of xenobiotic percutaneous absorption utilizing the isolated perfused porcine skin flap. J. Pharm. Sci. 79:305-311 (1990).

17. T. Yano, A. Nakagawa, M. Tsuji, and K. Noda. Skin permeation of various non-steroidal anti-inflammatory drugs in man. Life Sci. 39:1043-1050 (1986).

18. V. H. W. Mak, R. O. Potts, and R. H. Guy. Percutaneous penetration enhancement in vivo measured by attenuated total reflectance infrared spectroscopy. Pharm. Res. 7:835-841 (1990).

19. R. H. Guy, D. A. W. Bucks, J. R. McMaster, D. A. Villaflor, K. V. Roskos, R. S. Hinz, and H. I. Maibach. Kinetics of drug absorption across human skin in vivo. In B. Shroot and H. Schaefer (eds.), Skin Pharmacokinetics, Karger, Basel, 1987, pp. 70-76.

20. A. Rougier. Predictive measurement of in vivo percutaneous absorption. In R. C. Scott, R. H. Guy, and J. Hadgraft (eds.), Prediction of Percutaneous Penetration: Methods, Measurements, Modelling, IBC Technical Services, London, 1990, pp. 19-33.

21. D. W. Osborne. Computational methods for prodrug or drug analogue selection optimized for percutaneous delivery. In D. W. Osborne and A. H. Amann (eds.), Topical Drug Delivery Formulations, Marcel Dekker, New York, 1990, pp. 109-126.

22. (a) B. Berner and E. R. Cooper. Models of skin permeability. In A. F. Kydonieus and B. Berner (eds.), Transdermal Delivery of Drugs, Vol. 2, CRC Press, Boca Raton, FL, 1987, pp. 410-56. (b) B. Berner. Pharmacokinetics of skin penetration. In A. F. Kydonieus and B. Berner (eds.), Transdermal Delivery of Drugs, Vol. 2, CRC Press, Boca Raton, FL, 1987, pp. 133-148.

23. (a) J. Hadgraft. Mathematical models of skin absorption. In R. C. Scott, R. H. Guy, and J. Hadgraft (eds.), Prediction of Percutaneous Penetration: Methods, Measurements, Modelling, IBC Technical Services, Ltd., London, 1990, pp. 252-262. (b) J. N. McDougal, H. J. Clewell, M. E. Andersen, and G. W. Jepson. Physiologically based pharmacokinetic modelling of skin penetration. In R. C. Scott, R. H. Guy, and J. Hadgraft (eds.), Prediction of Percutaneous Penetration: Methods, Measurements, Modelling, IBC Technical Services, Ltd., London, 1990, pp. 263-272.

24. R. H. Guy, A. H. Guy, H. I. Maibach, and V. P. Shah. The bioavailability of dermatological and other topically administered drugs. Pharm. Res. 3:253-262 (1986).

25. D. Caron, C. Queille-Roussel, V. P. Shah, and H. Schaefer. Correlation between the drug penetration and the blanching effect of topically applied hydrocortisone creams in human beings. J. Am. Acad. Dermatol. 23:458-462 (1990).

26. B. W. Barry. Optimizing percutaneous absorption. In R. L. Bronaugh and H. I. Maibach (eds.), Percutaneous Absorption. Mechanisms-Methodology-Drug Delivery, 2nd ed., Marcel Dekker, New York, 1989, pp. 531-554.

27. C. Queille-Roussel, M. Poncet, and H. Schaefer. Quantification of skin color changes induced by topical corticosteroid preparations using the Minolta chromameter. Br. J. Dermatol. 124: 264-270 (1991). 